\title{
A supplemental device to return escaping particles to a magnetic mirror reactor
}

Mitsuaki Nagata ${ }^{1 *}$ and Keiichi Sawada ${ }^{2}$

\author{
* Correspondence: scc.kei@gmail. \\ com \\ ${ }^{1}$ Nippon Electronic Engineering \\ College, Noboribetsu-shi, Hokkaido, \\ Japan \\ Full list of author information is \\ available at the end of the article
}

\begin{abstract}
Cyclotron resonance is now applied as one of the important means for heating plasma in a fusion reactor. We examined this phenomenon from the viewpoint of electron gyration orbits through a solution of the linearized relativistic equation of motion. We found a powerful term that accelerates a relativistic charged particle largely at a resonance point when a magnetic field strength is very large. In this study, aiming an effect of this term, we consider applying a resonance phenomenon to reducing the number of charged particles that escape from a magnetic mirror reactor. We install a long supplemental device at the exit of a main magnetic bottle and make a cyclotron resonance space within the device, as shown in Fig. 7. If velocities (perpendicular to a magnetic field) of charged particles are accelerated largely within the cyclotron resonance space, the reflection efficiency of a magnetic mirror behind the resonance space ought to be improved. Based on this idea, we discuss such a supplemental device for recovering the maximum number of escaping charged particles.
\end{abstract}

Keywords: Magnetic mirror reactor, Cyclotron resonance, Acceleration of a relativistic charged particle, Suppression of escaping particles loss

\section{Introduction}

In the early days of cyclotrons, the study of cyclotron resonance served to determine gas plasma condition from the characteristics of electromagnetic waves having passed through the plasma [1] But large quantities of wave energy are absorbed by charged particles when the wave frequency is nearly equal to the particles' cyclotron frequency. This phenomenon is now an important means to heat charged particles in a gas plasma [2-7]. We examined cyclotron resonance from a viewpoint of gyration orbits of an electron in a plane perpendicular to a static magnetic field, using a solution of the linearized relativistic equation of motion which was obtained under the condition given in Eq. (5) later. In this examination, we found a term that accelerates a relativistic charged particle rapidly, when a magnetic field strength is very large, at a resonance point where an electric field frequency is equal to a charged particle's cyclotron frequency. The term is given as second part in Eq. (18). Aiming an effect of this term, we consider improving a reflection efficiency of a magnetic mirror by installing a resonance space within the magnetic mirror. Plasma confinement by a magnetic mirror was greatly expected in the early stages of fusion research [8-10]. This is because adiabatic theory with respect to a motion of a charged particle (a particle moves as it winds around one magnetic flux) gave a clear theoretical prediction for plasma confinement. But magnetic mirror-type reactors had the drawback that too many charged

(c) The Author(s); licensee Springer on behalf of EPJ. 2018 Open Access This article is distributed under the terms of the Creative Commons Attribution 4.0 International License (http://creativecommons.org/licenses/by/4.0/), which permits unrestricted use, distribution, and reproduction in any medium, provided you give appropriate credit to the original author(s) and the source, provide a link to the Creative Commons license, and indicate if changes were made. 
particles are lost along the magnetic field line. Both the open magnetic (mirror-type) and the closed magnetic (toroidal-type) systems still have instability problems that obstruct the way to a controlled fusion reactor. Mirror-type research continues in mirror hybrid reactors [11-13]. In hybrid reactor research, plasma confinement demands can be relaxed if the fission reaction induces strong energy multiplication. However, research reports that shortcomings still exist, such as the charged particle loss from the loss cone and associated low electron temperature.

To counter these two shortcomings, we consider a means of installing a supplemental device (which has a cyclotron resonance space within) at the exit of a main magnetic bottle. Rapidly accelerating charged particles within the cyclotron resonance space greatly reduces the half-vertical angle of the loss cone in a magnetic mirror behind the resonance space. Thus, in Section 2, we first derive a solution of the linearized relativistic equation of motion for an electron and the powerful term that appears in the resonance point. In Section 3, we discuss an effect of a long magnetic field configuration that extends outside a main bottle (shown in Fig. 7). That device has cyclotron resonance spaces (electric fields) within and is designed to reflect the maximum number of escaping charged particles.

\section{Gyration orbits of electrons}

We first complement the previous work [14] with respect to the linearization of the relativistic equation of motion:

$$
\frac{\partial}{\partial t}\left(\frac{m_{e}}{\left(1-\frac{v\left(t, t_{0}\right)^{2}}{c^{2}}\right)^{\frac{1}{2}}} \boldsymbol{v}\left(t, t_{0}\right)\right)=-q \mathbf{E}(t)-q \boldsymbol{v}\left(t, t_{0}\right) \times \mathbf{B} .
$$

Here, $m_{e}$ is the rest mass of an electron, $-q$ is the electron charge, $c$ is the speed of light, $\mathbf{E}(t)$ is an electric field, B is a magnetic field, and $\boldsymbol{v}\left(t, t_{0}\right)$ is the velocity of the electron at time $t$ after scattering with an initial velocity $\boldsymbol{v}_{0}\left(=\hat{x} v_{0 x}+\hat{y} v_{0 y}+\hat{z} v_{0 z}\right)$ from a heavy neutral atom at time $t_{0}$. Based on Eqs. (14)-(22) of the previous work [14]", Eq. (1) can be transformed into

$$
\begin{aligned}
& \left\{\left\{\frac{m_{e}}{\left(1-\frac{v_{0}^{2}}{c^{2}}\right)^{\frac{1}{2}}}+\int_{t_{0}}^{t} \frac{-q \mathbf{E}(t) \cdot \boldsymbol{v}\left(t, t_{0}\right)}{c^{2}} d t\right\} \frac{\partial \boldsymbol{v}\left(t, t_{0}\right)}{\partial t}+\boldsymbol{v}\left(t, t_{0}\right)\left(\frac{-q \mathbf{E}(t) \cdot \boldsymbol{v}\left(t, t_{0}\right)}{c^{2}}\right)\right. \\
& =-q \mathbf{E}(t)-q \boldsymbol{v}\left(t, t_{0}\right) \times \mathbf{B} .
\end{aligned}
$$

The physical meanings of the terms on the left-hand side of Eq. (2) are as follows:

(i) In the curly brackets \{\} , the quantity $m_{e} /\left(1-v_{0}^{2} / c^{2}\right)^{1 / 2}$ is the electron mass at time $t_{0}$. The integral $\int_{t_{0}}^{t}-q \mathbf{E}(t) \cdot v\left(t, t_{0}\right) d t / c^{2}$ is the inertial-mass increment from $t$ to $t_{0}$. The terms in the curly brackets sum to give the inertia mass at time $t$.

"The quantity $\alpha$ " in Eq. (21) of the previous work [14] should have been defined as $\int_{t_{0}}^{t}-q \mathbf{E}(t) \cdot \boldsymbol{v}\left(t, t_{0}\right) d t$, because it precedes the linearization.

(ii) The quantity $-q \mathbf{E}(t) \cdot \boldsymbol{v}\left(t, t_{0}\right) / c^{2}$ of the second term is the inertial-mass increment per unit time at time $t$.

(iii) The first term is the momentum increment per unit time coming from the increment in velocity $\partial v\left(t, t_{0}\right) / \partial t$. The second term is the momentum increment per unit time coming from the increment in inertial mass. 
Now, under the condition

$$
\frac{\left|\boldsymbol{v}\left(t, t_{0}\right)-\boldsymbol{v}_{t}\right|}{\left|\boldsymbol{v}_{t}\right|} \ll \cdot 1 \cdots \cdots \cdots\left(\boldsymbol{v}_{t}=\boldsymbol{v}\left(t, t_{0}\right)_{(\mathbf{E}(t)=0)}\right),
$$

Eq. (2) can be linearized as

$$
\begin{aligned}
& \frac{m_{e}}{\left(1-\frac{v_{0}^{2}}{c^{2}}\right)^{\frac{1}{2}}} \frac{\partial \boldsymbol{v}\left(t, t_{0}\right)}{\partial t}+\left(\int_{t_{0}}^{t} \frac{-q \mathbf{E}(t) \cdot \boldsymbol{v}_{t}}{c^{2}} d t\right) \frac{\partial \boldsymbol{v}_{t}}{\partial t}+\boldsymbol{v}_{t}\left(\frac{-q \mathbf{E}(t) \cdot \boldsymbol{v}_{t}}{c^{2}}\right) \\
& =-q \mathbf{E}(t)-q \boldsymbol{v}\left(t, t_{0}\right) \times \mathbf{B}
\end{aligned}
$$

We solve Eq. (4) for $\hat{z} \cdot \boldsymbol{v}\left(t, t_{0}\right)$ under the following external force fields:

$$
\begin{aligned}
& \mathbf{E}(t)=-\hat{z} \quad E \cos \omega t . \\
& \mathbf{B}=\hat{y} \quad B .
\end{aligned}
$$

To simplify, we use the following symbols:

$\boldsymbol{v}_{0}=\hat{x} v_{0 x}+\hat{y} v_{0 y}+\hat{z} v_{0 z} \equiv \hat{x} x_{0}+\hat{y} y_{0}+\hat{z} z_{0} \quad$ (The case where $x_{0}=0$ is treated hearafter),

$$
\begin{aligned}
& \frac{m_{e}}{\sqrt{1-\frac{v_{0}^{2}}{c^{\prime \prime}}}} \equiv m, \quad \frac{q B}{m_{e}} \sqrt{1-\frac{v_{0}^{2}}{c^{\prime \prime}}}=\frac{q B}{m} \equiv \omega_{c}, \\
& \boldsymbol{v}\left(t, t_{0}\right)=\hat{x} v_{x}\left(t, t_{0}\right)+\hat{y} v_{y}\left(t, t_{0}\right)+\hat{z} v_{z}\left(t, t_{0}\right) \equiv \hat{x} v_{x}^{\prime}+\hat{y} v_{y}^{\prime}+\hat{z} v_{z}^{\prime} \equiv \boldsymbol{v}^{\prime}, \\
& \cos \omega_{c}\left(t-t_{0}\right)=C, \sin \omega_{c}\left(t-t_{0}\right)=S, \cos \omega t=c, \quad \sin \omega t=s .
\end{aligned}
$$

In (6), note that $m$ is independent of time (but it is not the rest mass) and that $c$ is not the speed of light. As seen in the symbol $m$, the speed of light $c$ always appears in such forms as $\boldsymbol{v}_{0}^{2} / c^{2}$. To avoid confusion between the speed of light $c$ and the symbol $c=\cos \omega t$, from now we use $c$ " for the square of the speed of light. The four symbols $(C, S, c, s)$ are trigonometrical function, from now.

By using the following two relationships:

$$
\begin{aligned}
& \boldsymbol{v}_{t}=\boldsymbol{v}\left(t, t_{0}\right)_{(\mathbf{E}(t)=0)}=\hat{x}\left(z_{0} S\right)+\hat{y} y_{0}+\hat{z}\left(z_{0} C\right), \\
& \frac{\alpha^{\prime \prime}}{c^{\prime \prime}} \equiv \int_{t_{0}}^{t} \frac{-q \mathbf{E}(t) \cdot \boldsymbol{v}_{t}}{c^{\prime \prime}} d t=\frac{1}{c^{\prime \prime}} \frac{q E}{\omega_{c}^{2}-\omega^{2}}\left(z_{0}\left(\omega_{c} c S-\omega s C\right)+z_{0} \omega \sin \omega t_{0}\right),
\end{aligned}
$$

Eq. (4) takes the form

$$
\begin{aligned}
m \frac{\partial \mathbf{v}^{\prime}}{\partial t}+ & \frac{\alpha^{\prime \prime}}{c^{\prime \prime}}\left[\hat{x}\left(z_{0} \omega_{c} C\right)+\hat{z}\left(-z_{0} \omega_{c} S\right)\right] \\
& +\left[\hat{x}\left(z_{0} S\right)+\hat{y} y_{0}+\hat{z}\left(z_{0} C\right)\right] \frac{q E}{c^{\prime \prime}} C\left(z_{0} C\right) \\
& =\hat{z} q E c-q\left[\hat{x}\left(-v_{z}^{\prime} B\right)+\hat{z}\left(v_{z}^{\prime} B\right)\right]
\end{aligned}
$$

After eliminating $v_{x}^{\prime}$ from Eq. (9), we obtain the following second-order differential equation for $v_{z}^{\prime}$ : 


$$
\begin{aligned}
\frac{\partial^{2} v_{z}^{\prime}}{\partial t^{2}}+\omega_{c}^{2} v_{z}^{\prime}=-\frac{q E}{m} \omega S-A c S C\left[z_{0}^{2}\left(-6 \omega_{c}^{3}+4 \omega_{c} \omega^{2}\right)\right] \\
-A s C^{2}\left[z_{0}^{2} \omega\left(\omega_{c}^{2}+\omega^{2}\right)\right] \\
-A C\left[2 \omega_{c}^{2}\left(-z_{0}^{2} \omega \sin \omega t_{0}\right)\right],
\end{aligned}
$$

where,

$$
A=\frac{q E}{m c^{\prime \prime}} \frac{1}{\omega_{c}^{2}-\omega^{2}} .
$$

Solving Eq. (10) for $v_{z}^{\prime}$ under two initial conditions at $t=t_{0}$ which are given by

$$
\begin{array}{ll}
v_{z\left(t=t_{0}\right)}^{\prime} & =z_{0}, \\
\left(\frac{\partial v_{z}^{\prime}}{\partial t}\right)_{\left(t=t_{0}\right)} & =\frac{q E}{m} \cos \omega t_{0}-\frac{q E}{m c} z_{0}^{2} \cos \omega t_{0},
\end{array}
$$

we have

$$
v_{z}^{\prime}=a_{1} s+\left(a_{2} s\right) S^{2}+\left(a_{3} s\right) C^{2}+\left(a_{4} c\right) S C+a_{5} t S+C_{1} C+C_{2} S
$$

Here,

$$
\begin{aligned}
a_{1}= & \frac{q E}{m} \frac{-\omega}{\omega_{c}^{2}-\omega^{2}}, \\
a_{2}= & \frac{q E}{m c^{\prime \prime}} \frac{1}{\left(\omega_{c}^{2}-\omega^{2}\right)^{2}}\left[-2 \omega_{c}^{2} \omega z_{0}^{2}\right], \\
a_{3}= & \frac{q E}{m c^{\prime \prime}} \frac{1}{\left(\omega_{c}^{2}-\omega^{2}\right)^{2}}\left[\left(\omega_{c}^{2} \omega-\omega^{3}\right) z_{0}^{2}\right], \\
a_{4}= & \frac{q E}{m c^{\prime \prime}} \frac{2 \omega_{c}^{3}}{\left(\omega_{c}^{2}-\omega^{2}\right)^{2}}\left(-z_{0}^{2}\right), \\
a_{5}= & \frac{q E}{m c^{\prime \prime}} \frac{\omega_{c}}{\omega_{c}^{2}-\omega^{2}}\left(z_{0}^{2} \omega \sin \omega t_{0}\right), \\
C_{1}= & z_{0}-a_{1} \sin \omega t_{0}-a_{3} \sin \omega t_{0}, \\
C_{2}= & \frac{q E}{m \omega_{c}} \cos \omega t_{0}-\frac{q E}{m c^{\prime \prime}} \frac{1}{\omega_{c}} z_{0}^{2} \cos \omega t_{0}-a_{1} \frac{\omega}{\omega_{c}} \cos \omega t_{0} \\
& -a_{3} \frac{\omega}{\omega_{c}} \cos \omega t_{0}-a_{4} \cos \omega t_{0}-a_{5} t_{0} .
\end{aligned}
$$

We now examine electron orbits under the following simple condition:

$t_{0}=0$ (Then, the symbols S and C defined in Eq. (6) become $\sin \omega_{c} t$ and $\cos \omega_{c} t$, respectively. For these $\sin \omega_{c} t$ and $\cos \omega_{c} t$, we continue to use the same symbols $\mathrm{S}$ and $\mathrm{C}$, respectively)

In this case, we have

$$
\begin{aligned}
v_{z}^{\prime}=v_{z}(t)=z_{0} C & +\frac{q E}{m}\left(1-\frac{z_{0}^{2}}{c^{\prime \prime}}\right) \frac{1}{\omega_{c}} S+\frac{q E}{m} \frac{1}{\omega_{c}^{2}-\omega^{2}}\left(-\omega S+\frac{\omega^{2}}{\omega_{c}} S\right)+\frac{q E}{m} \frac{z_{0}^{2}}{c^{\prime \prime}} \frac{1}{\omega_{c}^{2}-\omega^{2}} \\
& \times\left(\omega S C^{2}-\frac{\omega^{2}}{\omega_{c}} S\right)+\frac{q E}{m} \frac{z_{0}^{2}}{c^{\prime \prime}} \frac{1}{\left(\omega_{c}^{2}-\omega^{2}\right)^{2}}\left[-2 \omega_{c}^{3}(c S C-S)-2 \omega_{c}^{2} \omega S S^{2}\right] .
\end{aligned}
$$

Also, under condition (13), we obtain from Eqs. (9) and (12) 


$$
\begin{aligned}
v_{x}^{\prime}=v_{x}(t) & =z_{0} S+\frac{q E}{m} c \frac{\omega_{c}}{\omega_{c}^{2}-\omega^{2}}+C\left[\frac{q E}{m} \frac{-\omega}{\omega_{c}^{2}-\omega^{2}}+\frac{q E}{m} \frac{z_{0}^{2}}{c^{\prime \prime}} \frac{-\omega_{c}\left(\omega_{c}^{2}+\omega^{2}\right)}{\left(\omega_{c}^{2}-\omega^{2}\right)^{2}}\right] \\
& +S C s\left[\frac{q E}{m} \frac{z_{0}^{2}}{c^{\prime \prime}} \frac{3 \omega_{c}^{2} \omega-\omega^{3}}{\left(\omega_{c}^{2}-\omega^{2}\right)^{2}}\right]+S^{2} c\left[\frac{q E}{m} \frac{z_{0}^{2}}{c^{\prime \prime}} \frac{-\omega_{c}}{\omega_{c}^{2}-\omega^{2}}\right]+C^{2} c\left[\frac{q E}{m} \frac{z_{0}^{2}}{c^{\prime \prime}} \frac{\omega_{c}\left(\omega_{c}^{2}+\omega^{2}\right)}{\left(\omega_{c}^{2}-\omega^{2}\right)^{2}}\right] .
\end{aligned}
$$

Both $v_{z}^{\prime}$ and $v_{x}^{\prime}$ contain the factor $\omega_{c}^{2}-\omega^{2}$ in their denominators, so we find their values in the limit $\omega \rightarrow \omega_{c}$ (It should be noted that $\omega_{c}$ is independent of time):

$$
\begin{aligned}
& v_{z r} \equiv \lim _{\omega \rightarrow \omega_{c}} v_{z}(t)=z_{0} C+\frac{q E}{m}\left(1-\frac{z_{0}^{2}}{c^{\prime \prime}}\right) \frac{1}{\omega_{c}} S+\frac{q E}{m}\left(\frac{1}{-2 \omega_{c}}\left(S-\omega_{c} t C\right)\right)+\frac{q E}{m} \frac{z_{0}^{2}}{c^{\prime \prime}} \\
& \times\left(\frac{1}{8 \omega_{c}^{2}}\left(2 \omega_{c}^{3} t^{2} S-4 \omega_{c}^{2} t C+4 \omega_{c} S+6 \omega_{c} S^{3}\right)\right) \\
&= z_{0} C+\frac{1}{2} \frac{q E}{m \omega_{c}}\left(1-\frac{z_{0}^{2}}{c^{\prime \prime}}\right) S+\frac{1}{2} \frac{q E}{m} t\left(1-\frac{z_{0}^{2}}{c^{\prime \prime}}\right) C+\frac{1}{4} \frac{q E}{m \omega_{c}} \frac{z_{0}^{2}}{c^{\prime \prime}}\left(\omega_{c}^{2} t^{2}+3 S^{2}\right) S . \\
& v_{x r} \equiv \lim _{\omega \rightarrow \omega_{c}} v_{x}(t)=z_{0} S+\frac{1}{2} \frac{q E}{m} t S+\frac{q E}{m} \frac{z_{0}^{2}}{c^{\prime \prime}}\left(\frac{1}{8 \omega_{c}^{2}}\left(-4 \omega_{c}^{2} t S-2 \omega_{c}^{3} t^{2} C-6 \omega_{c} S^{2} C\right)\right) \\
&=z_{0} S+\frac{1}{2} \frac{q E}{m} t\left(1-\frac{z_{0}^{2}}{c^{\prime \prime}}\right) S-\frac{1}{4} \frac{q E}{m \omega} \frac{z_{0}^{2}}{c^{\prime \prime}}\left(\omega_{c}^{2} t^{1}+3 S^{2}\right) C . \\
& v_{r} \equiv\left(v_{z r}^{2}+v_{x r}^{2}\right)^{\frac{1}{2}} \simeq\left\{\left[z_{0}+\frac{(-q)(-E)}{2 m} t\left(1-\frac{z_{0}^{2}}{c^{\prime \prime}}\right)\right]^{2}+\left[\frac{(-q)(-E) t}{m} \cdot \frac{z_{0}^{2}}{c^{\prime \prime}} \cdot \frac{\omega_{c} t}{4}\right]^{2}\right\}^{1 / 2} \\
& \quad \text { (first part) }
\end{aligned}
$$

Furthermore, in the limit $\omega_{c} \rightarrow 0, \quad v_{z r}$ and $v_{x r}$ become, respectively

$$
\begin{aligned}
& \lim _{\omega_{c} \rightarrow 0} v_{z r}=z_{0}+\frac{q E}{m} t\left(1-\frac{z_{0}^{2}}{c^{\prime \prime}}\right) \\
& \lim _{\omega_{c} \rightarrow 0} v_{x r}=0 .
\end{aligned}
$$

When an electric field is weak, as seen in Eq. (17) of ref. [14], only the terms of the zero-order and the first-order with respect to an electric field are commonly held theoretically. In Eq. (18), however, the new term (the second part) of the second-order with respect to an electric field has appeared. From the examination of the characteristics shown in "from Figs. 1a-d, 2, 3, 4, 5, 6, 8, 9, 10, 11 and 12 (except Fig. 7)" which are drawn based on Eqs. (12), (20) and (21), the second part works clearly to increase a velocity-magnitude (perpendicular to a magnetic field) of a relativistic electron, when $\omega_{c} t \gg 1$. Asking for a limit at the resonance point from Eq. (17) of ref. [14], under the following initial condition:

At $t=t_{0}=0$, an initial velocity $\boldsymbol{v}_{0}=\hat{z} v_{0 z}\left(\left|\boldsymbol{v}_{0}\right|=v_{0}\right)$, we have

$\lim _{\omega \rightarrow \omega_{c r}\left(v_{0}\right)}($ Eq. (17) of ref. [14] $)=v_{0 z}+\gamma_{r}\left(v_{0}\right)^{3} \frac{q E_{0 z}}{2 m_{e}}\left(t+\frac{\sin \omega_{c r}\left(v_{0}\right) t}{\omega_{c r}\left(v_{0}\right)} \cos \omega_{c r}\left(v_{0}\right) t\right)$.

(we have now used the same symbols with in ref. [14]).

When $\omega_{c r}\left(v_{0}\right) t \gg 1$, (where $\omega_{c r}\left(v_{0}\right)$ is the same quantity with $\omega_{c}$ given in Eq. (6)), the above expression is the same one with Eq. (18) without the second part. 


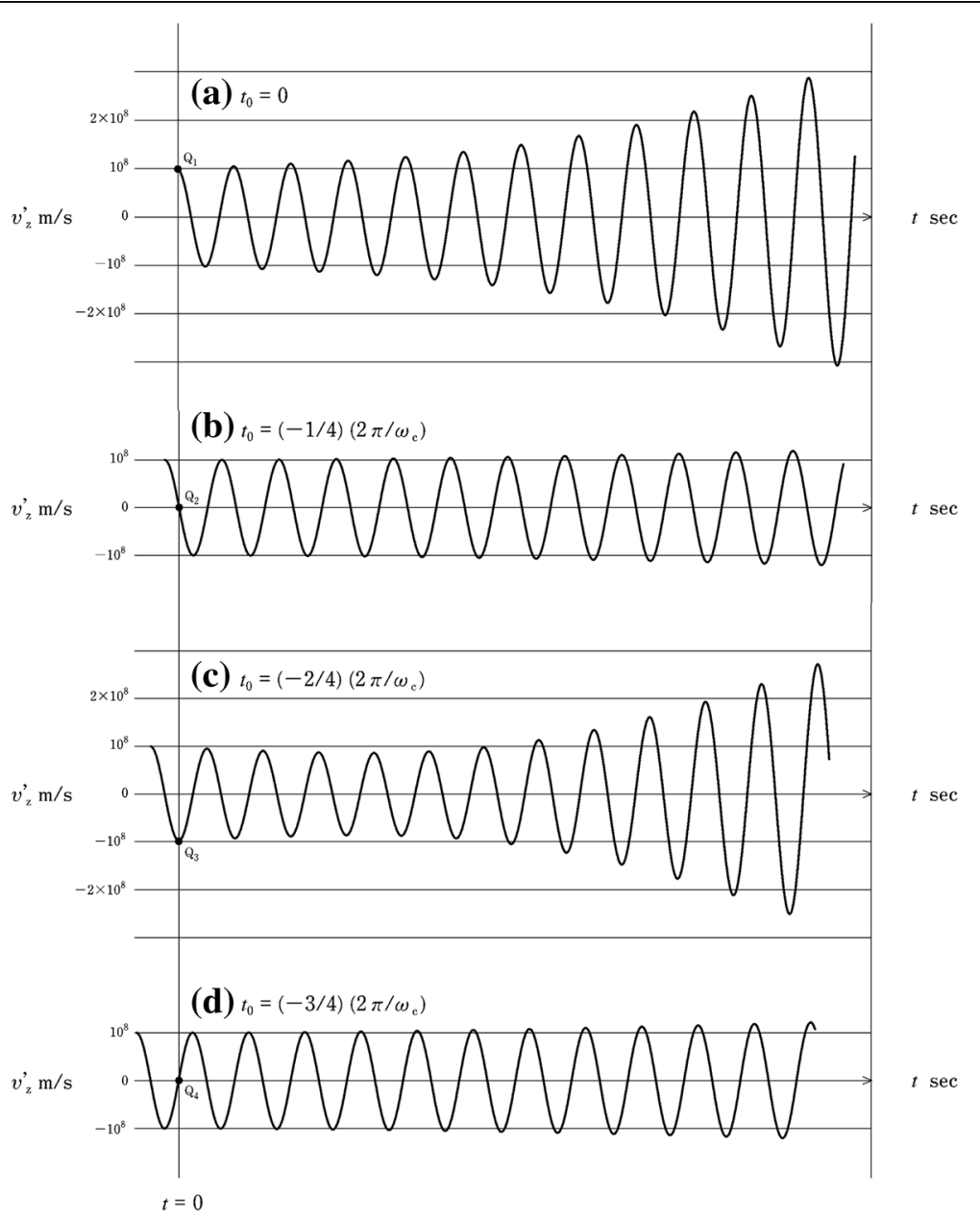

Fig. 1 a-d Dependence of a velocity $u_{z}^{\prime}(z$ - component) of a relativistic electron on the phase of an electric field, based on Eq. (12). The physical conditions are: an initial velocity $z_{0}=\left|\hat{z} 10^{8}\right| \mathrm{m} / \mathrm{s}$ at time $t_{0}$ (written in each of $(\mathbf{a})-(\mathbf{d})), \mathbf{E}(t)=-\hat{z} 10^{3} \cos \omega t \mathrm{~V} / \mathrm{m}, \omega_{c}=q|\hat{y} \mathbf{B}|\left(1-z_{0}^{2} / c^{\prime \prime}\right)^{1 / 2} / m_{e}=10^{8}\left(1-z_{0}^{2} / c^{\prime \prime}\right)^{1 / 2} \mathrm{sec}^{-1}$, and $\omega_{c} / \omega=1.0001$. The flight time $t=t_{0} \sim t_{0}+12\left(2 \pi / \omega_{c}\right) \mathrm{sec}$

We now ask for the coordinates $(z(t), x(t))$ of an electron based on Eqs. (14) and (15). The electron starts at the origin $(x=z=0)$ with an initial velocity of $\hat{z} z_{0}\left(=\hat{z} v_{0 z}\right)$ at time $t=0$. The results are:

$$
\begin{aligned}
z(t) & =\int_{0}^{t} v_{z}(t) d t=z_{0} \frac{\sin \omega_{c} t}{\omega_{c}}+\frac{q E}{m} \frac{-\omega}{\omega_{c}^{2}-\omega^{2}} \frac{1-\cos \omega t}{\omega}(20) \\
& +\frac{q E}{m} \frac{z_{0}^{2}}{c^{\prime \prime}} \frac{-2 \omega_{c}^{2} \omega}{\left(\omega_{c}^{2}-\omega^{2}\right)^{2}}\left(\frac{1}{4}\right)\left[\left(\frac{\cos \left(2 \omega_{c}+\omega\right) t}{2 \omega_{c}+\omega}-\frac{\cos \left(2 \omega_{c}-\omega\right) t}{2 \omega_{c}-\omega}-\frac{2 \cos \omega t}{\omega}\right)-\left(\frac{1}{2 \omega_{c}+\omega}-\frac{1}{2 \omega_{c}-\omega}-\frac{2}{\omega}\right)\right] \\
& +\frac{q E}{m} \frac{z_{0}^{2}}{c^{\prime \prime}} \frac{\omega}{\omega_{c}^{2}-\omega^{2}}\left(\frac{-1}{4}\right)\left[\left(\frac{\cos \left(2 \omega_{c}+\omega\right) t}{2 \omega_{c}+\omega}-\frac{\cos \left(2 \omega_{c}-\omega\right) t}{2 \omega_{c}-\omega}+\frac{2 \cos \omega t}{\omega}\right)-\left(\frac{1}{2 \omega_{c}+\omega}-\frac{1}{2 \omega_{c}-\omega}+\frac{2}{\omega}\right)\right] \\
& +\frac{q E}{m} \frac{z_{0}^{2}}{c^{\prime \prime}} \frac{-2 \omega_{c}^{3}}{\left(\omega_{c}^{2}-\omega^{2}\right)^{2}}\left(\frac{-1}{4}\right)\left[\left(\frac{\cos \left(2 \omega_{c}+\omega\right) t}{2 \omega_{c}+\omega}+\frac{\cos \left(2 \omega_{c}-\omega\right) t}{2 \omega_{c}-\omega}\right)-\left(\frac{1}{2 \omega_{c}+\omega}+\frac{1}{2 \omega_{c}-\omega}\right)\right] \\
& +\left(\frac{q E}{m} \frac{\omega_{c}}{\omega_{c}^{2}-\omega^{2}}+\frac{q E}{m} \frac{z_{0}^{2}}{c^{\prime \prime}} \frac{\omega_{c}\left(\omega_{c}^{2}+\omega^{2}\right)}{\left(\omega_{c}^{2}-\omega^{2}\right)^{2}}\right) \frac{1-\cos \omega_{c} t}{\omega_{c}}
\end{aligned}
$$




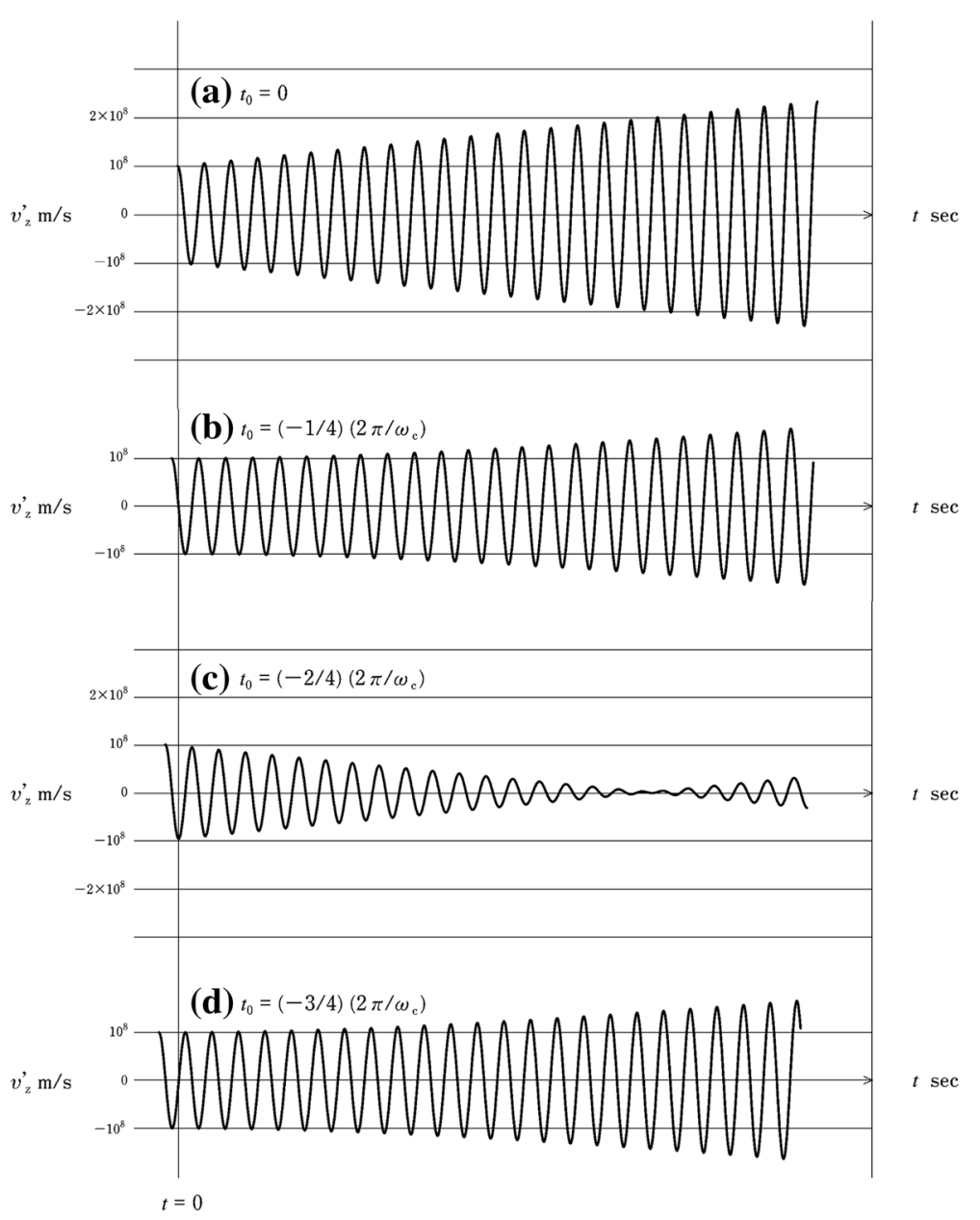

Fig. 2 a-d Dependence of a velocity $U_{z}^{\prime}$ ( $z$ - component) of a nonrelativistic electron on the phase of an electric field. The conditions are the same with in Fig. 1a-d, except for the following two changes:

$\left\{c^{\prime \prime}=\left(3 \times 10^{8} \mathrm{~m} / \mathrm{sec}\right)^{2} \rightarrow\left(3 \times 10^{8} \times 10^{6} \mathrm{~m} / \mathrm{sec}\right)^{2}\right.$

$\left\{t=t_{0} \sim t_{0}+12\left(2 \pi / \omega_{c}\right) \rightarrow t=t_{0} \sim t_{0}+24\left(2 \pi / \omega_{c}\right)\right.$ sec Every relativistic effect in Eq. (12) is acompanied

by the factor $z_{0}^{2} / c^{\prime \prime}$. So, we obtain nonrelativistic characteristics by setting $c^{\prime \prime}$ to $\left(3 \times 10^{8} \times 10^{6} \mathrm{~m} / \mathrm{sec}\right)^{2}$, from now

$$
\begin{aligned}
x(t)= & \int_{0}^{t} v_{x}(t) d t=z_{0} \frac{1-\cos \omega_{c} t}{\omega_{c}}+\frac{q E}{m} \frac{\omega_{c}}{\omega_{c}^{2}-\omega^{2}} \frac{\sin \omega t}{\omega} \\
& +\frac{q E}{m} \frac{-\omega_{c}}{\omega_{c}^{2}-\omega^{2}}\left(1+\frac{\omega_{c}^{2}+\omega^{2}}{\omega_{c}^{2}-\omega^{2}} \frac{z_{0}^{2}}{c^{\prime \prime}}\right) \frac{\sin \omega_{c} t}{\omega_{c}}+\frac{q E}{m} \frac{z_{0}^{2}}{c^{\prime \prime}} \frac{3 \omega_{c}^{2} \omega-\omega^{3}}{\left(\omega_{c}^{2}-\omega^{2}\right)^{2}}\left(\frac{-1}{4}\right)\left[\frac{\sin \left(2 \omega_{c}+\omega\right) t}{2 \omega_{c}+\omega}-\frac{\sin \left(2 \omega_{c}-\omega\right) t}{2 \omega_{c}-\omega}\right] \\
& +\frac{q E}{m} \frac{z_{0}^{2}}{c^{\prime \prime}} \frac{\omega_{c}\left(\omega_{c}^{2}+\omega^{2}\right)}{\left(\omega_{c}^{2}-\omega^{2}\right)^{2}}\left(\frac{-1}{4}\right)\left[\frac{\sin \left(2 \omega_{c}+\omega\right) t}{2 \omega_{c}+\omega}+\frac{\sin \left(2 \omega_{c}-\omega\right) t}{2 \omega_{c}+\omega}+\frac{2 \sin \omega t}{\omega}\right] \\
& +\frac{q E}{m} \frac{z_{0}^{2}}{c^{\prime \prime}} \frac{\omega_{c}\left(\omega_{c}^{2}+\omega^{2}\right)}{\left(\omega_{c}^{2}-\omega^{2}\right)^{2}}\left(\frac{1}{4}\right)\left[\frac{\sin \left(2 \omega_{c}+\omega\right) t}{2 \omega_{c}+\omega}+\frac{\sin \left(2 \omega_{c}-\omega\right) t}{2 \omega_{c}-\omega}+\frac{2 \sin \omega t}{\omega}\right] .
\end{aligned}
$$

In the case where $\omega_{c} / \omega=1.0001$, we examine dependences of Eq. (12) on the phase of an electric field. The results are shown in Figs. 1a-d and 2a-d. An electron starts at the origin with an initial velocity $+\hat{z} 10^{8} \mathrm{~m} / \mathrm{s}$ at four different phases of time $t_{0}(0,(-1 / 4)(2 \pi$ $\left./ \omega_{c}\right),(-2 / 4)\left(2 \pi / \omega_{c}\right),(-3 / 4)\left(2 \pi / \omega_{c}\right)$, where $2 \pi / \omega_{c}(\simeq 2 \pi / \omega)$ is almost the period of the 


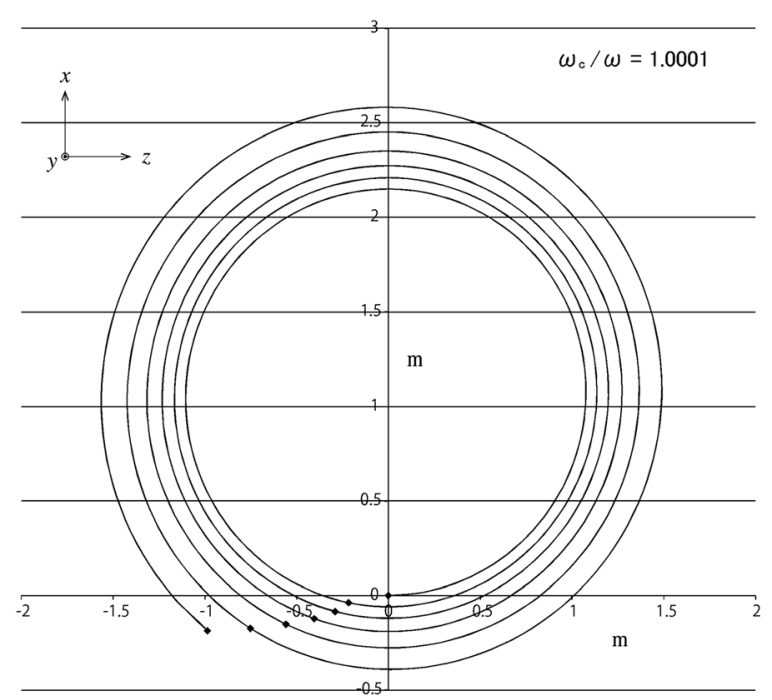

Fig. 3 The gyration orbit of a relativistic electron based on Eqs. (20) and (21). The initial velocity $\hat{z} z_{0}=\hat{z} 10^{8} \mathrm{~m} / \mathrm{s}$ at $(t=0), \mathbf{E}(t)=-\hat{z} 10^{3} \cos \omega t \mathrm{~V} / \mathrm{m}, \omega_{c}=q|\hat{y} \mathbf{B}|\left(1-z_{0}^{2} / c^{\prime \prime}\right)^{1 / 2} / m_{e}=10^{8}\left(1-z_{0}^{2} / c^{\prime \prime}\right)^{1 / 2} \mathrm{sec}^{-1}$. The flight time $t=0 \sim 6\left(2 \pi / \omega_{c}\right)$ sec. An electron rotates about six times. The dots mark each time increment of $2 \pi / \omega_{c}$ sec. This figure is related with Fig. 1 a

electric field). We set flight times $t$ as "from $t_{0}$ to $t_{0}+12\left(2 \pi / \omega_{c}\right)$ " for a relativistic electron and "from $t_{0}$ to $t_{0}+24\left(2 \pi / \omega_{c}\right)$ " for a nonrelativistic electron. In the cases where $t_{0}=0$ and $t_{0}=(-2 / 4)\left(2 \pi / \omega_{c}\right)$, or in Fig. 1a and c, electrons are accelerated very rapidly when $\omega_{c} t \gg 1$. On the other hand, in the cases of Fig. $1 \mathrm{~b}$ and d, electrons are hardly accelerated. From points $\mathrm{Q}_{1}, \mathrm{Q}_{2}, \mathrm{Q}_{3}$ and $\mathrm{Q}_{4}$ in Fig. 1a-d, we can obtain approximate values of velocities near $t=0$. Since a gyration period of an electron is about $2 \pi / \omega_{c}$, each velocity at points $\mathrm{Q}_{1}, \mathrm{Q}_{2}$, $\mathrm{Q}_{3}$ and $\mathrm{Q}_{4}$ is given nearly by

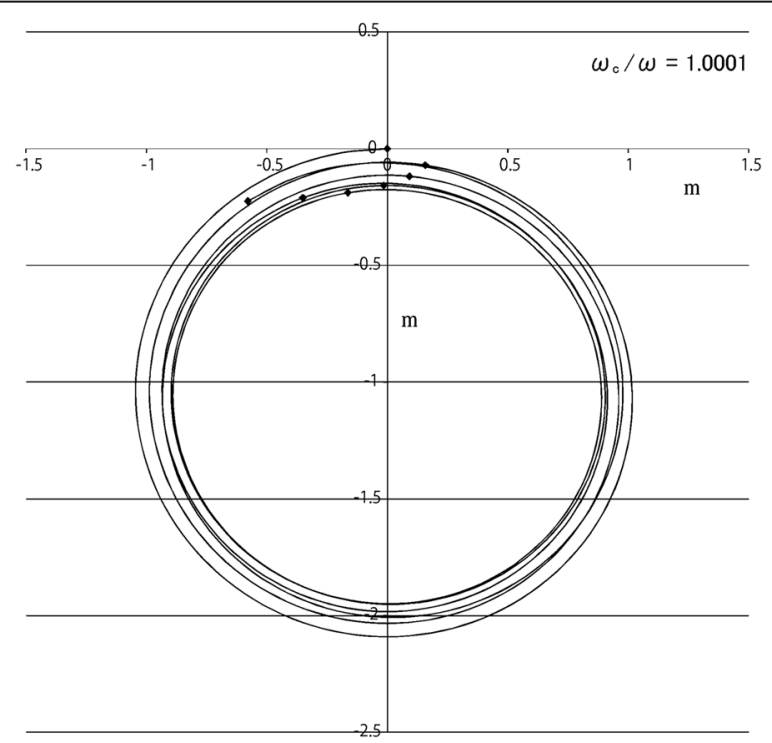

Fig. 4 The gyration orbit of a relativistic electron. The conditions are the same with in Fig. 3, except for the following change of the initial velocity at $(t=0): \hat{z} z_{0}=\hat{z} 10^{8} \rightarrow-\hat{z} 10^{8} \mathrm{~m} / \mathrm{s}$. This figure is related with Fig. $1 \mathrm{c}$ 


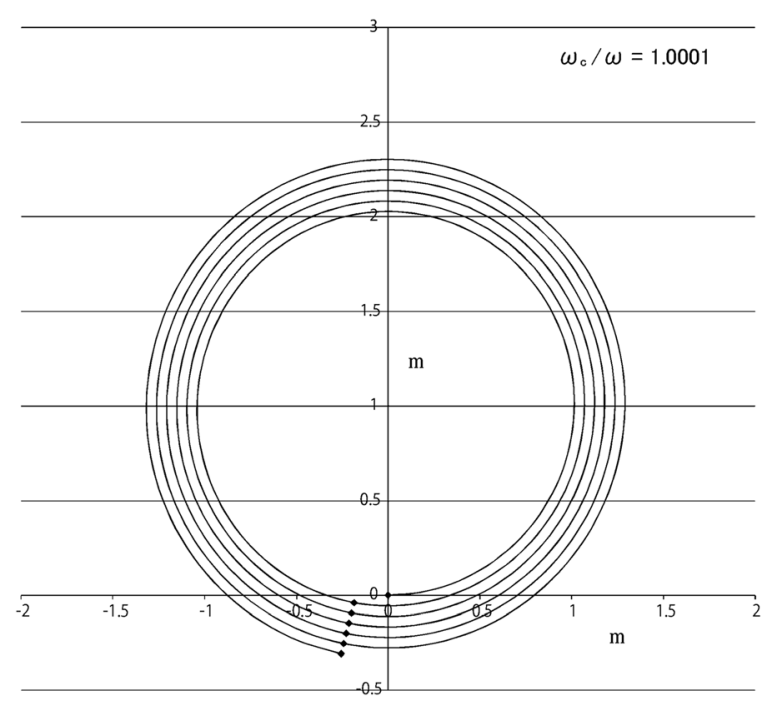

Fig. 5 The gyration orbit of a nonrelativistic electron. The conditions are the same with in Fig. 3, except for the following change: $c^{\prime \prime}=\left(3 \times 10^{8} \mathrm{~m} / \mathrm{sec}\right)^{2} \rightarrow\left(3 \times 10^{8} \times 10^{6} \mathrm{~m} / \mathrm{sec}\right)^{2}$ This figure is related with Fig. $2 \mathrm{a}$
$\left(\hat{\mathrm{z}} 10^{8}+\hat{\mathrm{x}} 0\right) \mathrm{m} / \mathrm{s}$
at $\mathrm{Q}_{1}(t=0)$ (the exact value only at $\mathrm{Q}_{1}$ ),
$\left(\hat{\mathrm{z}} 0+\hat{\mathrm{x}} 10^{8}\right) \mathrm{m} / \mathrm{s}$
at $\mathrm{Q}_{2}(t \simeq 0)$,
$\left(\hat{\mathrm{z}}\left(-10^{8}\right)+\hat{\mathrm{x}} 0\right) \mathrm{m} / \mathrm{s}$
at $\mathrm{Q}_{3}(t \simeq 0)$,
$\left(\hat{\mathrm{z}} 0+\hat{\mathrm{x}}\left(-10^{8}\right)\right) \mathrm{m} / \mathrm{s}$
at $\mathrm{Q}_{4}(t \simeq 0)$.

Accordingly, electrons starting with $\pm \hat{z} 10^{8} \mathrm{~m} / \mathrm{s}$ into the electric field space at $t \simeq 0$ are largely accelerated, but electrons starting with $\pm \hat{x} 10^{8} \mathrm{~m} / \mathrm{s}$ at $t \simeq 0$ are hardly accelerated. In the nonrelativistic cases of Fig. $2 a-d$, it is seen that electrons are accelerated

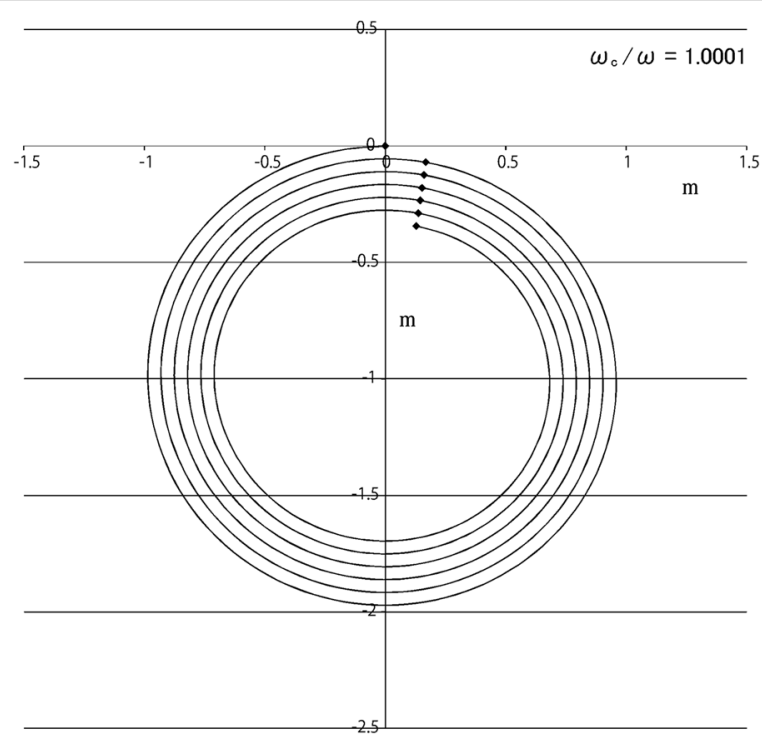

Fig. 6 The gyration orbit of a nonrelativistic electron. The conditions are the same with in Fig. 3, except for the following two changes: $\left\{\begin{array}{l}c^{\prime \prime}=\left(3 \times 10^{8} \mathrm{~m} / \mathrm{sec}\right)^{2} \rightarrow\left(3 \times 10^{8} \times 10^{6} \mathrm{~m} / \mathrm{sec}\right)^{2} \\ \text { The initial velocity at }(t=0) \quad \hat{z} z_{0}=\hat{z} 10^{8} \rightarrow-\hat{z} 10^{8} \mathrm{~m} / \mathrm{s} .\end{array}\right.$ This figure is related with Fig. 2(c) 


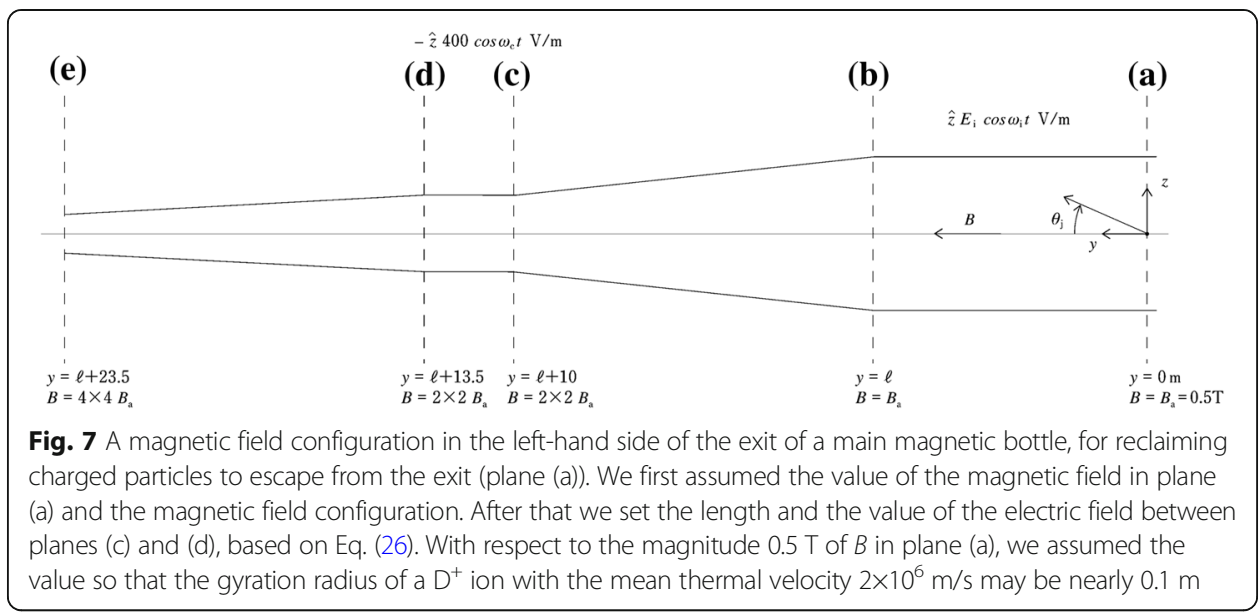

slowly by the electric field, but the acceleration pace is very slow compared with the relativistic cases of Fig. 1a and c.

According to the variation in Fig. 1a, an electron seems to be able to obtain a velocity-magnitude over the speed of light after longer flight time. Such is due to break of the linearization condition in Eq. (3). If an exact speed of the electron in Fig. 1a increases as a function of time through most of its flight time, the mass increment per unit time $-q \mathbf{E}(t) \cdot \boldsymbol{v}_{\boldsymbol{t}} / c^{\prime \prime}$ based on Eq. 3 is less than the exact increment $-q \mathbf{E}(t) \cdot \boldsymbol{v}\left(t, t_{0}\right)$ / $c$. When an electron gyrates as it grows heavier than is in the case in Fig. 1a, the relativistic effect increasing the speed will be reduced compared with that in Fig. 1a. The rate of increase of the velocity-magnitude in Fig. 1a ought to reduce to zero before the speed of the electron goes beyond the speed of light.

Next, we show in Figs. 3, 4, 5 and 6 gyration orbits which are drawn based on Eqs. (20) and (21). A resonance point is set to $\omega_{c} / \omega=1.0001$. We described numerical conditions in Figs. 3, 4, 5 and 6. The orbit for a nonrelativistic electron in Fig. 6 seems to contract according to the first part $-\left|z_{0}\right|+q E t /\left(2 m_{e}\right)$ of Eq. (18). Conversely, the orbit for a relativistic electron in Fig. 4 initially contracts, but soon begins a rapid expansion. We consider that the cause of this reversal is due to the second part of Eq. (18). In Section 3, based on Eq. (18), we try devising a supplemental magnetic mirror having electric fields within to reflect escaping relativistic electrons to an old magnetic bottle.

\section{Discussion of a supplemental device in a mirror type reactor}

We now discuss a supplemental device for reducing the rate at which charged particles escape a magnetic mirror reactor. We consider a magnetic field configuration such as shown in Fig. 7, at the exit of a magnetic bottle. The magnetic field tail is divided into four sections by five planes labeled (a), (b), (c), (d) and (e) in Fig. 7. We define the $x, y$, $z$ coordinate-system as shown in Fig. 7. The $y$-coordinate and magnetic field $B$ at each plane are also given in Fig. 7. We regard the magnetic field $B$ to be only in $+y$-direction. An electric field $+\hat{z} E_{i} \cos \omega_{i} t\left(\omega_{i}\right.$; ion cyclotron frequency) between planes (a) (b), and $-\hat{z} 400 \cos \omega_{c} t$ between planes (c) - (d) are supplied.

We assume that a plasma density in the magnetic field tail is considerably more tenuous than plasma densities within magnetic mirror bottles or tokamak reactors [15-20] 
and so the influence of Coulomb forces on the orbits of charged particles is negligible within the magnetic field tail.

The condition $[21,22]$ for a continuous $\mathrm{D}-\mathrm{D}$ reaction $\left(\mathrm{D}={ }_{1} 2 \mathrm{H}\right)$ is commonly estimated to be that the $\mathrm{D}^{+}$temperature exceeds $4 \times 10^{8} \mathrm{~K}$. The thermal velocity of an electron at $4 \times$ $10^{8} \mathrm{~K}$ is about $1.2 \times 10^{8} \mathrm{~m} / \mathrm{s}(=\bar{v})$. To simplify the discussion from now, we assume that every electron escaping through plane (a) from the main bottle has the velocity $\bar{v}$. We denote an initial velocity of an electron when passing plane $(j)(j=\mathrm{a}, \mathrm{b}, \ldots, \mathrm{e})$ by

$$
\hat{x} x_{0(j)}+\hat{y} y_{0(j)}+\hat{z} z_{0(j)}
$$

and denote by $v_{r(j)}$ a velocity-magnitude perpendicular to $B$ of an electron when passing plane $(j)$. Also, for an incident angle with which a charged particle passes plane ( $j$ ), we denote an angle from $+y$-axis in the $y$ - $z$ plane by $\theta_{j}\left(-90^{\circ} \leqq \theta_{j} \leqq 90^{\circ}\right)$ and a mere inclination from $+y$-axis by $|\theta|_{j}\left(0^{\circ} \leqq|\theta|_{j} \leqq 90^{\circ}\right)$.

Now, let us aim electrons passing plane (c) at time $t=0$ with four kinds of velocities, $\pm \hat{z} z_{0(c)}+\hat{y} y_{0(c)}$ and $\pm \hat{x} x_{0(c)}+\hat{y} y_{0(c)}$ (where $z_{0(c)}=x_{0(c)}$, and these electrons are called $e_{z}^{ \pm}, e_{x}^{ \pm}$, respectively). It is mentioned later that $y_{0(c)}$ hardly change within space (c) (d). From the characteristics in Figs. $1 \mathrm{a}-\mathrm{d}$ and $8 \mathrm{a}-\mathrm{d}$ shown later, it is expected that $e_{z}^{ \pm}$ are largely accelerated by the electric field $-\hat{z} 400 \cos \omega t\left(\omega_{c} / \omega=1.0001\right)$ within space (c) - (d) but $e_{x}^{ \pm}$are hardly accelerated. Then, we first consider increasing the velocitymagnitude perpendicular to $B$ of $e_{z}^{-}$within space (c) - (d) so that $e_{z}^{-}$may be reflected by mirror $(\mathrm{d})-(\mathrm{e})$. We call $e_{z}^{-}$the test electron.

Here, we calculate $v_{y}^{\prime}\left(=v_{y}\left(t, t_{0}\right)\right)$ from the $y$-component of Eq.(9):

$$
\begin{aligned}
v_{y}^{\prime} & =v_{y}\left(t, t_{0}\right) \\
& =y_{0}+y_{0} z_{0} \frac{q E}{m c^{\prime \prime}}\left\{\frac{\sin \left[\left(\omega_{c}+\omega\right)\left(t-t_{0}\right)\right]}{2\left(\omega_{c}+\omega\right)}+\frac{\sin \left[\left(\omega_{c}-\omega\right)\left(t-t_{0}\right)\right]}{2\left(\omega_{c}-\omega\right)}\right\} .
\end{aligned}
$$

Also, when $t_{0}=0$, we have

$$
\lim _{\omega \rightarrow \omega_{c}} v_{y}^{\prime}=y_{0}+y_{0} z_{0} \frac{q E t}{2 m c^{\prime \prime}}\left\{1+\frac{\sin 2 \omega_{c} t}{2 \omega_{c} t}\right\} .
$$

The second term of Eq. (23) is compared with $y_{0}$ later. In the case where there is no electric field in the supplemental device of Fig. 7, let us ask for a half-vertical angle of the loss cone (the loss angle) in mirror (c)-(e) with respect to the test electron. The condition ratio for the test electron to be reflected is given by

$$
\frac{v_{r(c)}}{\left(v_{r(c)}^{2}+y_{0(c)}^{2}\right)^{1 / 2}}>\left(\frac{2 \times 2 B_{a}}{4 \times 4 B_{a}}\right)^{1 / 2} \quad \text { or } \quad v_{r(c)}>0.58 y_{0(c)},
$$

where, $2 \times 2 B_{a}$ and $4 \times 4 B_{a}$ are the magnetic fields in planes (c), (e), respectively, $v_{r(c)}=\left|z_{0(c)}\right|=\left|\bar{v} \sin \theta_{c}\right|, \quad y_{0(c)}=\bar{v} \cos \theta_{c}$ and $\theta_{c}<0$. When $\theta_{c}=-30^{\circ}$,

$$
\begin{aligned}
& v_{r(c)}=\left|z_{0(c)}\right|=0.6 \times 10^{8} \mathrm{~m} / \mathrm{s}, \\
& 0.58 y_{0(c)}=0.6 \times 10^{8} \mathrm{~m} / \mathrm{s} .
\end{aligned}
$$

Accordingly, the loss angle of mirror (c)-(e) for the test electron is $30^{\circ}$. 


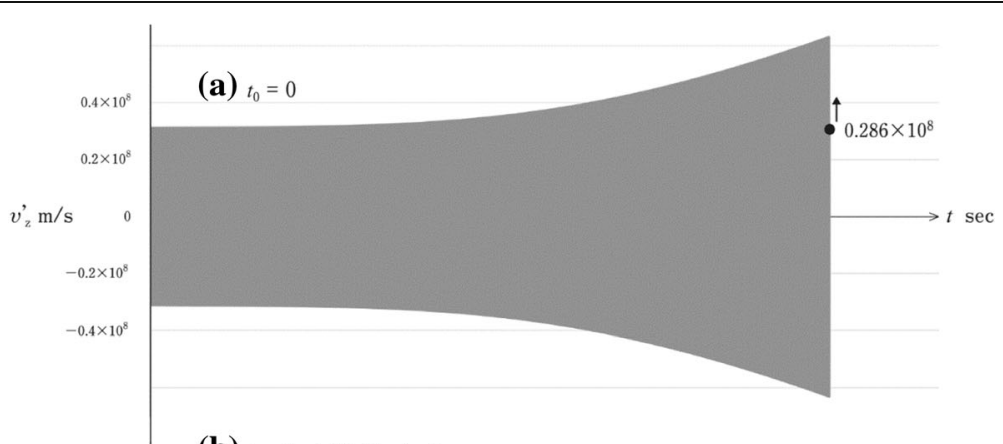

(b) $t_{0}=(-1 / 4)\left(2 \pi / \omega_{\mathrm{c}}\right)$

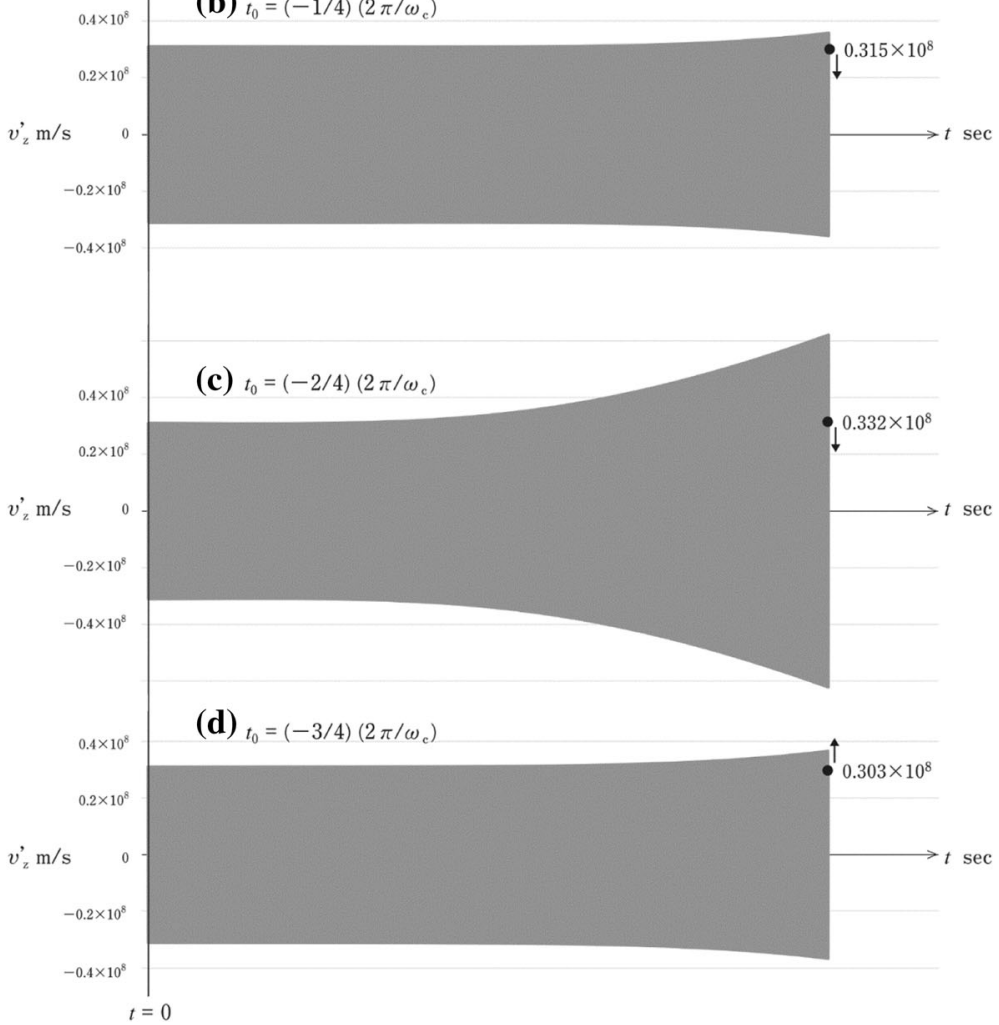

Fig. 8 a-d Dependence of a velocity $u_{z}^{\prime}$ of a relativistic electron on the phase of an electric field, based on Eq. (12). The figures are drawn under the same physical conditions with for Eq. (27): an initial velocity $z_{0}=\left|\hat{z}\left(-0.31 \times 10^{8}\right)\right| \mathrm{m} / \mathrm{s}$ at time $t_{0}, \mathbf{E}(t)=-\hat{z} 400 \cos \omega t \mathrm{~V} / \mathrm{m}, \omega_{c}=\left|\hat{y} q \mathbf{B} / m_{e}\right|\left(1-z_{0}^{2} / c^{\prime \prime}\right)^{1 / 2}=\mid \hat{y} 3.5$ $\times 10^{11} \mid \mathrm{sec}^{-1}, \omega_{c} / \omega=1.0001$. The flight time $t=t_{0} \sim t_{0}+\left(2 \pi / \omega_{c}\right) n=t_{0}+3 \times 10^{-8} \sec$ when $n=1670$. The dots mark the last velocity points after the flight

Now, we decrease the loss angle of mirror (c)-(e) for the test electron from $30^{\circ}$ to $15^{\circ}$ by using an electric field $-\hat{z} 400 \cos \omega_{c} t$ between planes (c) and (d). Let us recover by mirror (d)-(e) the test electron passing plane (c) at $t=0$ with

$$
\left\{\begin{array}{l}
\theta_{c}=-15^{\circ}, \\
z_{0(c)}=1.2 \times 10^{8} \sin \left(-15^{\circ}\right)=-0.31 \times 10^{8} \mathrm{~m} / \mathrm{s}, \\
y_{0(c)}=1.2 \times 10^{8} \cos \left(-15^{\circ}\right)=1.16 \times 10^{8} \mathrm{~m} / \mathrm{s}, \\
x_{0(c)}=0 .
\end{array}\right.
$$

Then, a velocity-magnitude $v_{r(d)}$ perpendicular to the magnetic field $B$ of the test electron when passing plane (d) must satisfy 


$$
v_{r(d)}>0.58 y_{0(c)}=0.67 \times 10^{8} \mathrm{~m} / \mathrm{s}
$$

because a reflection condition of mirror (d) - (e) is given by the replacement $v_{r(c)} \rightarrow$ $v_{r(d)}$ in Eq. (24). The electric field $-\hat{z} 400 \cos \omega_{c} t$ must accelerate the velocitymagnitude perpendicular to $B$ of the test electron from $\left|z_{0(c)}\right|=0.31 \times 10^{8} \mathrm{~m} / \mathrm{s}$ to $v_{r(d)}=0.67 \times 10^{8} \mathrm{~m} / \mathrm{s}$ as shown in Eq. (25). We estimate $v_{r(d)}$ using the following approximation of Eq. (18):

$$
v_{r} \simeq\left\{\left[z_{0}+\frac{q E t}{2 m_{e}}\right]^{2}+\left[\frac{q E t}{m_{e}} \frac{z_{0}^{2}}{c^{\prime \prime}} \frac{\omega_{c} t}{4}\right]^{2}\right\}^{\frac{1}{2}} \quad\left(\omega_{c} t \simeq q B t / m_{e} \gg 1\right) .
$$

Substituting

$$
\begin{aligned}
\frac{q}{m_{e}} & =1.76 \times 10^{11} \mathrm{C} / \mathrm{kg}, \quad E=400 \mathrm{~V} / \mathrm{m}, \quad B=0.5 \times 4=2 \mathrm{~T}, \quad t=\frac{3.5}{y_{0(c)}} \simeq 3 \times 10^{-8} \mathrm{~s} \\
z_{0} & =z_{0(c)}=-0.31 \times 10^{8} \mathrm{~m} / \mathrm{s}
\end{aligned}
$$

into Eq. (26), we have

$$
v_{r}=0.67 \times 10^{8} \mathrm{~m} / \mathrm{s} \quad\left(\omega_{c} t=1.06 \times 10^{4} \gg 1\right) .
$$

This $v_{r}$ satisfies the condition of Eq. (25). We have also tried drawing an electron's gyration orbit based on Eqs. (20) and (21) under the same numerical conditions as for Eq. (27). The result is shown in Fig. 9. When the gyration number $n$ is 1670, or when the gyration time is $\left(2 \pi / \omega_{c}\right) \times 1670\left(=3 \times 10^{-8} \mathrm{sec}\right)$, the gyration radius is approximately two times compared with the one of the initial circle. We estimate that the velocitymagnitude perpendicular to $B$ has also grown by about a factor of two. If the exact rate of increase of a velocity-magnitude in a relativistic orbit of an electron is smaller than the rate of increase shown in Fig. 9, then, the necessary gyration time to obtain $v_{r}=$ $0.67 \times 10^{8} \mathrm{~m} / \mathrm{s}$ of Eq. (25) becomes longer. In an actual design, however, this point can be solved by adjusting the value of $E$ or $t$. We also note that, as $q E t / 2 m_{e} \simeq 1.06 \times 10^{6}$ $\mathrm{m} / \mathrm{s}$ in Fig. 9, the second term of Eq. (23) is negligible compared with $y_{0}\left(=y_{0(c)}\right)$. We add also three Figs. 10, 11 and 12 in which a part of the numerical conditions in Fig. 9 are changed. We see that the pace at which an electron gains energy is very slow in a nonrelativistic condition.

Now, even if we had decreased the loss angle only for the test electron from $30^{\circ}$ to $15^{\circ}$ as mentioned above, more than a half of electrons having $|\theta|_{c}$ equal to $15^{\circ}$ pass through mirror (d) - (e), which is presumed from the characteristics of Fig. 8a-d. However, since magnitudes of $v_{z}^{\prime}$ in Fig. 8a-d still continue to increase with time, let us adjust the value $E t^{2}$ in the second part of Eq. (26) so that mirror (c) - (e) may reflect $74 \%$ of electrons with $|\theta|_{c}$ between $30^{\circ} \sim 15^{\circ}$. Then, since the loss rate of electrons is nearly proportional to the square of the loss angle (small angle), the loss angle of mirror (c) (e) becomes $20^{\circ}$ from the estimation of the ratio $\left(30^{2}-20^{2}\right) /\left(30^{2}-15^{2}\right)$. If mirror (c) (e) can reflect $79.9 \%$ of electrons with $|\theta|_{c}$ between $30^{\circ} \sim 15^{\circ}$, then, the loss angle of mirror (c) - (e) becomes $19^{\circ}$.

Under the above design, we consider the loss angle of mirror (a) - (e). An electron or a $\mathrm{D}^{+}$ion actually interacts only with the electric field having $\omega_{c}$ or $\omega_{i}$,respectively. The loss angles for electrons of mirror (d) - (e), mirror (b) - (c) and mirror (a) - (e) 


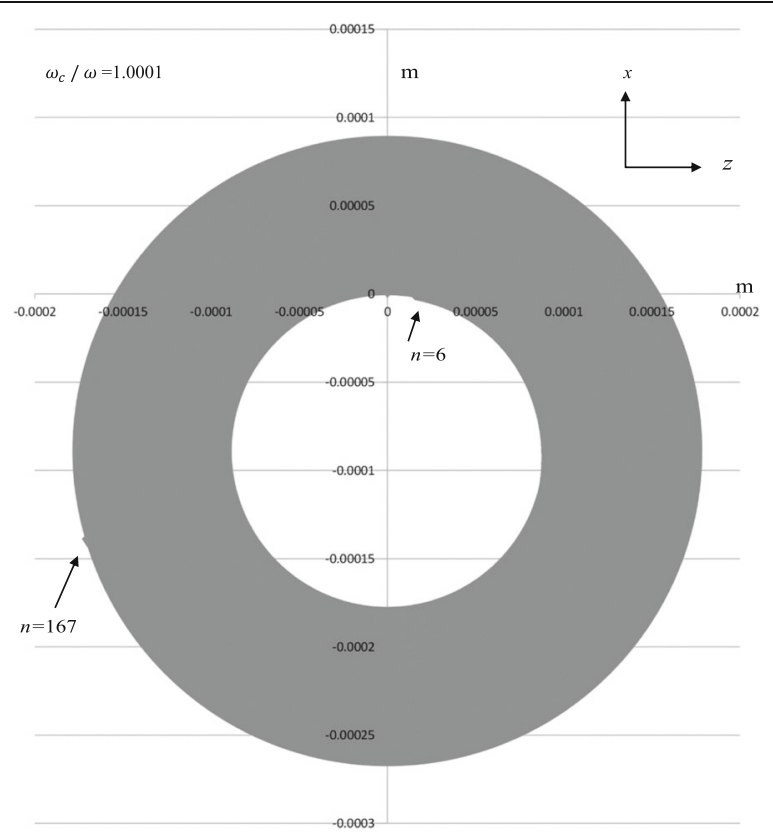

Fig. 9 The gyration orbit of a relativistic electron in the $x-z$ plane, based on Eqs. (20) and (21). The initial velocity $\hat{z} z_{0}=-\hat{z} 0.31 \times 10^{8} \mathrm{~m} / \mathrm{s}$ at $(t=0), \mathbf{E}(t)=-\hat{z} 400 \cos \omega t, B=2.0 \mathrm{~T}, \omega_{c}=\left|\hat{y} 3.5 \times 10^{11}\right| \mathrm{sec}^{-1}$ and the flight time $t=0 \sim\left(2 \pi / \omega_{c}\right) n=3 \times 10^{-8} \mathrm{sec}$ when $n=1670$. This figure is related with Fig. $8 \mathrm{c}$

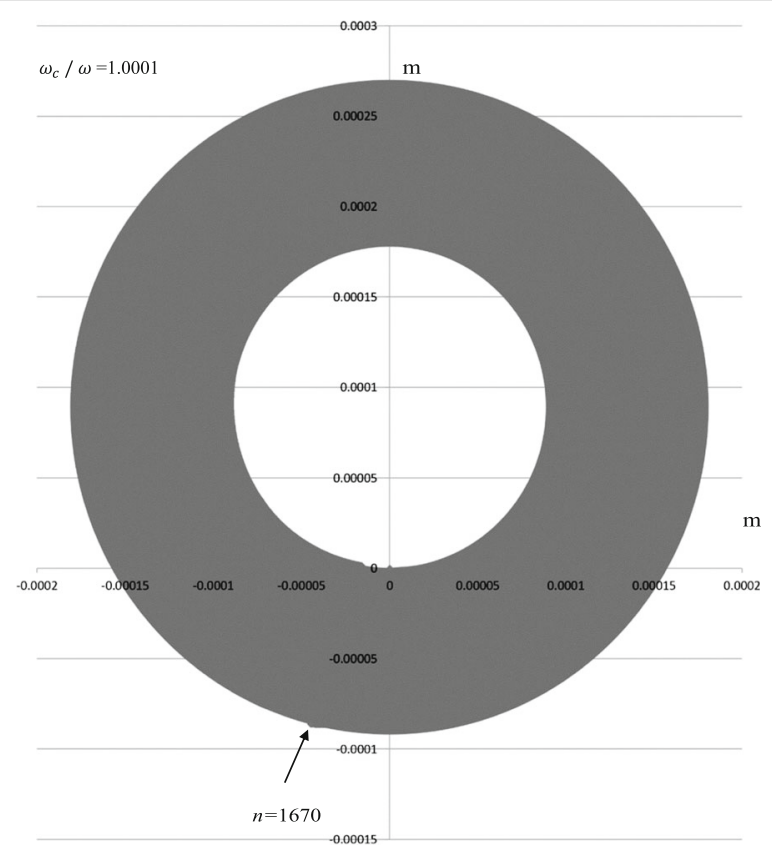

Fig. 10 The gyration orbit of a relativistic electron in the $x-z$ plane. The physical conditions are the same with in Fig. 9, except for the following change of an initial velocity at $(t=0): \hat{z} z_{0}=-\hat{z} 0.31 \times 10^{8} \rightarrow+\hat{z} 0.3$ $1 \times 10^{8} \mathrm{~m} / \mathrm{s}$. This figure is related with Fig. $8 \mathrm{a}$ 


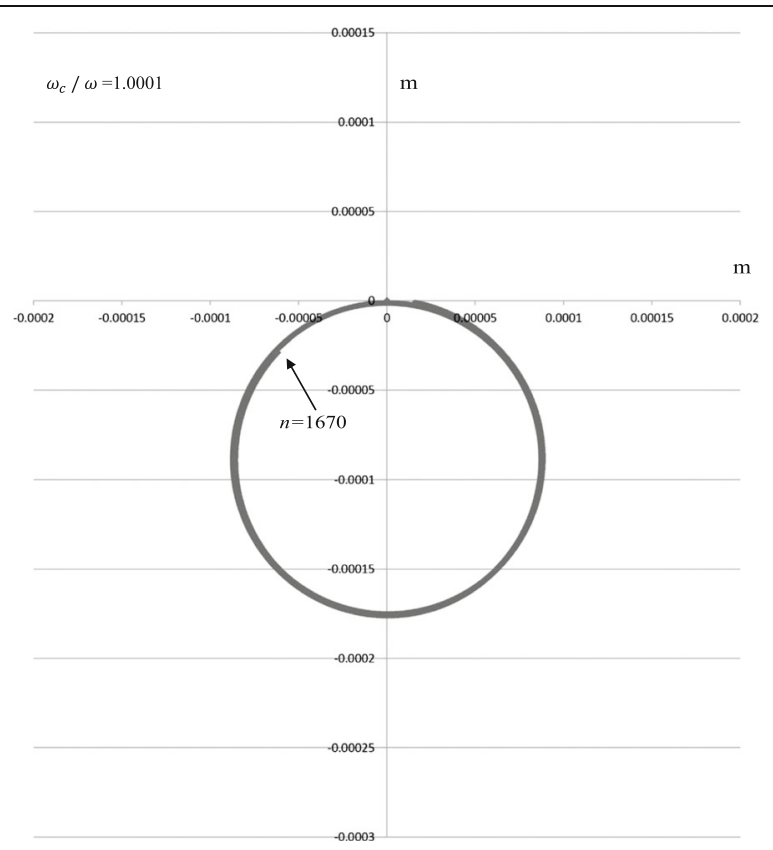

Fig. 11 The gyration orbit of a nonrelativistic electron. The physical conditions are the same with in Fig. 9, except for the following change: $c^{\prime \prime}=\left(3 \times 10^{8} \mathrm{~m} / \mathrm{sec}\right)^{2} \rightarrow\left(3 \times 10^{8} \times 10^{6} \mathrm{~m} / \mathrm{sec}\right)^{2}$

(without the electric field) are $30^{\circ}, 30^{\circ}$ and $15^{\circ}$, respectively. Accordingly, with respect to electrons, dependences of $|\theta|_{j}$ on $|\theta|_{a}$ become as follows:

(i) without the help of the electric field between planes (c) and (d),

For $|\theta|_{a}=30^{\circ} ;|\theta|_{c}=90^{\circ}$.

For $|\theta|_{a}=15^{\circ} ;|\theta|_{c}=30^{\circ},|\theta|_{e}=90^{\circ}$.

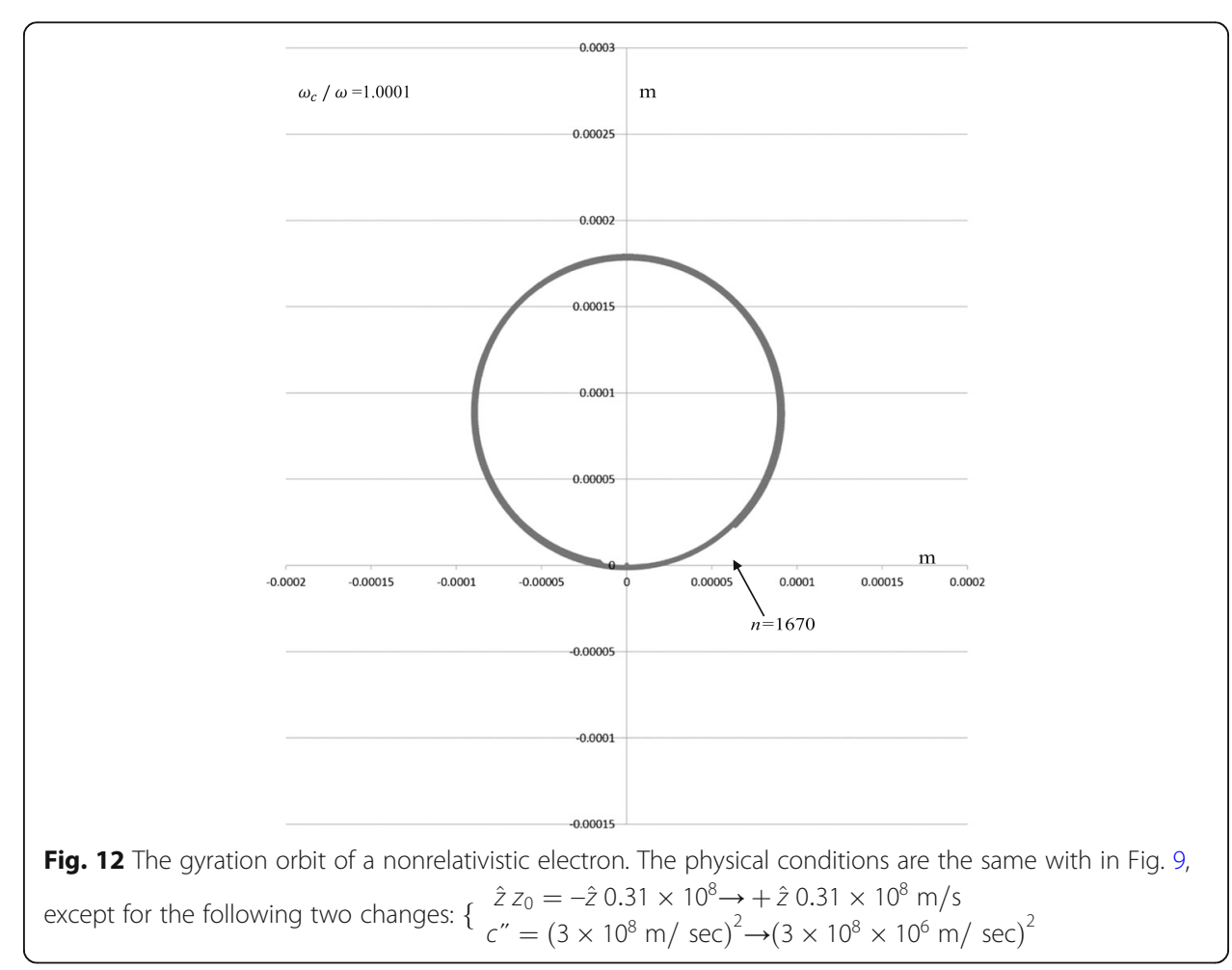


(ii) with the help of the electric field between planes (c) and (d) (the loss angle $19^{\circ}$ of mirror (c) - (e)),

$$
\begin{aligned}
& \text { For }|\theta|_{a}=15^{\circ} ;|\theta|_{c}=30^{\circ},|\theta|_{\text {between }} d-e=90^{\circ} . \\
& \text { For }|\theta|_{a} \simeq 10^{\circ} ; \quad|\theta|_{c}=19^{\circ}, \quad|\theta|_{e}=90^{\circ} .
\end{aligned}
$$

The supplemental device reflects escaping electrons whose angles $|\theta|_{a}$ are between $90^{\circ}$ and about $10^{\circ}$. The electric field improves the loss angle by about $5^{\circ}$. In order to decrease furthermore the loss angle of the supplemental device from $10^{\circ}$ to $10^{\circ}-\delta^{\circ}$, it is considered to set a new resonance space just in the right-hand side of plane (b). A new space must be devised so that electrons escaping with $\left(|\theta|_{a}=\left(10^{\circ}-\delta^{\circ}\right)\right)$ in plane (a) may have $\left(|\theta|_{b}=10^{\circ}\right)$ in plane (b).

Next, we ask for the loss angle of mirror (a)-(e) for $\mathrm{D}^{+}$ions (mass $m_{i}=3680 m_{e}$ ). A mean thermal velocity of $\mathrm{D}^{+}$ions at $4 \times 10^{8} \mathrm{~K}$ is about

$$
1.2 \times 10^{8}\left(m_{e} / m_{i}\right)^{1 / 2}=2 \times 10^{6} \mathrm{~m} / \mathrm{s} \equiv \bar{v}_{i} .
$$

Since $\bar{v}_{i}^{2} / \mathrm{c}^{\prime \prime}=4.4 \times 10^{-5}$, a $\mathrm{D}^{+}$ion is a nonrelativistic particle and the relativistic term for $\mathrm{a}^{+}$ion corresponding to the second part of Eq. (26) can be neglected compared to the term for a $\mathrm{D}^{+}$ion corresponding to the first term of Eq. (26). In the same manner as the treatment of electrons, we assume that every $\mathrm{D}^{+}$ions escaping from the main bottle has the velocity $\bar{v}_{i}$. We aim a $\mathrm{D}^{+}$ion passing plane (a) with a negative angle in the $y-z$ plane (called the test ion). From the characteristics in Fig. $2 \mathrm{a}-\mathrm{d}$ for an electron, if we can make the test ion reflect by mirror (a) - (e) having $\hat{z} E_{i} \cos \omega_{i} t$ between planes (a) and (b), we presume that mirror (a) - (e) reflects most of $\mathrm{D}^{+}$ions having the same inclination with $|\theta|_{a}$ of the test ion in plane (a). An initial velocity of the test ion when passing plane (a) is given by

$$
\hat{y} \bar{v}_{i} \cos \theta_{a}+\hat{z} \bar{v}_{i} \sin \theta_{a}+\hat{x} 0\left(\text { where }-90^{\circ}<\theta_{a}<0^{\circ}\right) .
$$

In the case of no electric field, a reflection condition of mirror (a) - (e) for the test ion is

$$
\frac{v_{r i}}{\left(v_{r i^{2}}+\left[\bar{v}_{i} \cos \theta_{a}\right]^{2}\right)^{\frac{1}{2}}}>\left(\frac{B_{a}}{4 \times 4 B_{a}}\right)^{\frac{1}{2}} \quad \text { or } \quad v_{r i}>0.26 \bar{v}_{i} \cos \theta_{a}
$$

where $v_{r i}$ is a velocity-magnitude perpendicular to $B$ of the test ion when passing plane (b). Now, in the case of $\theta_{a}=-15^{\circ}$, inserting.

$$
\begin{gathered}
\hat{\mathrm{z}} \bar{v}_{i} \sin \left(-15^{\circ}\right)=2 \times 10^{6} \times(-0.259)=-0.52 \times 10^{6} \mathrm{~m} / \mathrm{s} . \\
\left(v_{r i} \text { is }\left|\hat{\mathrm{z}} \bar{v}_{i} \sin \left(-15^{\circ}\right)\right| \text { in the case of no electric field }\right), \\
\hat{\mathrm{y}} \bar{v}_{i} \cos \left(-15^{\circ}\right)=2 \times 10^{6} \times 0.966=1.92 \times 10^{6} \mathrm{~m} / \mathrm{s} .
\end{gathered}
$$$$
\text { into Eq. (29), we have. }
$$$$
v_{r i}\left(=\left|\bar{v}_{i} \sin \left(-15^{\circ}\right)\right|=0.52 \times 10^{6}\right)>0.26 \bar{v}_{i} \cos \left(-15^{\circ}\right)=0.5 \times 10^{6} \mathrm{~m} / \mathrm{s} \text {. }
$$

Accordingly, the loss angle of mirror (a)-(e) for the test ion is regarded to be about $15^{\circ}$. Now, supplying electric field $+\hat{z} E_{i} \cos \omega_{i} t$ between planes (a) and (b), we consider decreasing the loss angle for the test ion from $15^{\circ}$ to $10^{\circ}$. The test ion passing plane (a) with the angle of $\theta_{a}=-10^{\circ}$ has the velocity.

$$
\hat{\mathrm{z}} \bar{v}_{i} \sin \left(-10^{\circ}\right)+\hat{\mathrm{y}} \bar{v}_{i} \cos \left(-10^{\circ}\right)+\hat{\mathrm{x}} 0=\hat{\mathrm{z}}\left(-0.35 \times 10^{6}\right)+\hat{\mathrm{y}} 1.97 \times 10^{6} \mathrm{~m} / \mathrm{s} \text {. }
$$

Accordingly, the electric field must accelerate the velocity $\left|\hat{\mathrm{z}} \bar{v}_{i} \sin \left(-10^{\circ}\right)\right|$ to $0.26 \bar{v}_{i}$ $\cos \left(-10^{\circ}\right)\left(=0.51 \times 10^{6} \mathrm{~m} / \mathrm{s}\right.$, from Eq. (29)). We estimate $v_{r i}$ from the following expression for $\mathrm{D}^{+}$ions corresponding to the first part of Eq. (26): 


$$
v_{r i} \simeq\left|\bar{v}_{i} \sin \theta_{a}+\frac{q E_{i} t}{2 m_{i}}\right| \quad\left(\omega_{i} t=q B t / m_{i} \gg 1\right)
$$

where $t=\ell / \bar{v}_{i} \cos \theta_{a}$ and $q / m_{i}=4.8 \times 10^{7} \mathrm{C} / \mathrm{kg}$.

The condition (29), $v_{r i}>0.26 \bar{v}_{i} \cos \theta_{a}\left(\theta_{a}=-10^{\circ}\right)$, is written as

$$
\left|-0.35 \times 10^{6}+\frac{4.8 \times 10^{7}}{2} E_{i} t\right|>0.51 \times 10^{6} \quad \text { or } E_{i} t>0.036 .
$$

Thus, we obtain

$$
\begin{array}{llll}
\text { (1) For } E_{i}=10^{3} \mathrm{~V} / \mathrm{m} ; & t=3.6 \times 10^{-5} \mathrm{~s}, \quad \ell=71 \mathrm{~m}, & \left(\omega_{i} t=864\right) . \\
\text { (2) For } E_{i}=10^{4} \mathrm{~V} / \mathrm{m} ; & t=3.6 \times 10^{-6} \mathrm{~s}, \quad \ell=7.1 \mathrm{~m}, & \left(\omega_{i} t=86.4\right) .
\end{array}
$$

We consider it possible to suppress the escaping number of electrons to a sufficiently small level. However, when the second part of Eq. (18) is ineffective by the reason that a particle is a nonrelativistic one, it has become clear that, in order to control massive and nonrelativistic $\mathrm{D}^{+}$ion by such a magnetic mirror as shown in Fig. 7 , an extremely powerful electric field is necessary.

\section{Conclusion}

For stable confinement of a gas plasma, it is important to keep electrical neutrality of a gas. Therefore, such a supplemental magnetic mirror as in Fig. 7 must have the same value of loss angle both for electrons and for ions. However, from the examination in Section 3 , confining $\mathrm{D}^{+}$ions will be the more difficult challenge. As a more realistic approach than producing an extremely powerful high-frequency electric field, we consider the following: (1) We first suppress the escaping number of $\mathrm{D}^{+}$ions as much as possible. (2) For the loss of still uncontrolled $\mathrm{D}^{+}$ions, we replenish $\mathrm{D}^{+}$ions from an external plasma source into the space between planes (d) and (e). In magnetic mirror experiments, a general method for plasma production is to create a weakly ionized plasma in advance and inject high energy charged particles from outside. The DCX device at Oak Ridge National Laboratory [10] created a gas plasma by injecting a 600-keV beam of $\mathrm{H}_{2}^{+}$ions. We could apply that technical method as a means for replenishing escaping $\mathrm{D}^{+}$ions.

Finally, we note that the supplemental device heats the plasma in the main bottle because both electrons and $\mathrm{D}^{+}$ions, which return to plane (a) after having approached plane (e), gain energy twice unrelated to the phases of the electric fields.

\section{Acknowledgements}

We are sincerely grateful to Chief Chika Adachi of the Heian Light Technology Company for her valuable advice on this study.

Funding

Nothing.

Availability of data and materials

No objection.

\section{Authors' contributions}

MN designed the study, and wrote the initial draft of the manuscript. KS contributed to analysis and interpretation of data, and assisted in the preparation of the manuscript. Both authors read and approved the final manuscript. 
Consent for publication

We agree to "Consent for publication".

\section{Competing interests}

The authors declare that they have no competing interests with respect to financial and non-finantial matters.

\section{Publisher's Note}

Springer Nature remains neutral with regard to jurisdictional claims in published maps and institutional affiliations.

\section{Author details}

${ }^{1}$ Nippon Electronic Engineering College, Noboribetsu-shi, Hokkaido, Japan. ${ }^{2}$ Soft Creator Company, Shinmachi,

Nakagyo-ku, Kyoto, Japan.

Received: 11 August 2017 Accepted: 17 January 2018

Published online: 09 February 2018

\section{References}

1. Kelly DC, Margenau H, Brown SC (1957) Phys Rev 108:1367

Artemyev AV, Agapitov OV, Krasnoselskikh W (2013) Phys Plasmas 20:124502

Caliri C, Mascali D, Volpe FA (2015) Nucl Instrum Methods A 790:57

4. Gospodchikov ED, Smolyakova OB (2015) Radiophys Quantum Electron 57:857

5. Ivanov AA, Burdakov AV, Bagryansky PA (2015) Fusion Sci Technol 68:56

6. Moiseenko VE, Ågren O (2012) AIP Conf Proc 1442:199

7. Fidone I, Granata G, Ramponi G, Meyer RL (1978) Phys Fluids 21(4):645

8. Gibson G, Jordan WC, Lauer EJ (1960) Phy Rev Lett 5:141

9. Linhart JG (1961) Plasma phys. Chap. 2. North-Holland Publishing Co., Netherlands

10. Krall NA, Trivelpiece AW (1973) Principles of plasma phys. Chap. 2. McGraw-Hill, USA

11. Ågren O, Moiseenko VE, Noack K, Hagnestal A (2011) Probl At Sci Technol 1:3

12. Ågren O, Moiseenko VE (2014) Plasma Phys Control Fusion 56:095026

13. Moir RW, Martovetsky NN, Molvik AW, Ryutov DD, Simonen TC (2012) Fusion Sci Technol 61:206

14. Nagata M (1990) Aust J Plant Physiol 44:47

15. Fisch NJ (1987) Rev Mod Phys 59:175

16. C. E. Myers, M. R. Edwards, B. Berlinger, A. Brooks, and S. A. Cohen, Princeton plasma physics laboratory report PPPL-4587 (2011)

17. Harvey RW, Nevins WM, Smith GR, Lloyd B, O’Brien MR, Warrick CD (1997) Nucl Fusion 37(1):69

18. A. J. Fetterman and N. J. Fisch, Princeton plasma physics laboratory report PPPL-4627 (2011)

19. Freidberg JP, Mangiarotti FJ, Minervini J (2015) Phys Plasmas 22:070901

20. Fredrickson ED, Bell MG, Budny RV, Darrow DS, White R (2015) Phys Plasmas 22:032501

21. K. Goto, Plasma Physics (the text printed in 1967), Kyoritu Publishing Co. Jpn

22. Lawson JD (1957) Proc Phys Soc B70:6

\section{Submit your manuscript to a SpringerOpen ${ }^{\circ}$ journal and benefit from:}

- Convenient online submission

- Rigorous peer review

- Open access: articles freely available online

- High visibility within the field

- Retaining the copyright to your article 